\title{
Pseudoconvex Domains on a Kähler Manifold with Positive Holomorphic Bisectional Curvature"
}

By

\author{
Osamu SUZUKI*
}

\section{§ 1. Introduction}

Let $M$ be an $n$-dimensional complex manifold with a real analytic kähler metric. Throughout this paper, a kähler manifold is assumed to have a real analytic kähler metric without mentioning it. A relatively compact domain $D$ in $M$ is called a pseudoconvex(resp. strongly pseudoconvex) domain if there exist a neighborhood $U$ of $p$ and a pseudoconvex (resp. strongly pseudoconvex) function $\varphi$ on $U$ satisfying $D \cap U=\{\varphi<0\}$ for each boundary point $p \in \partial D$. We write simply $s$-pseudoconvex domains (resp. functions) for strongly pseudoconvex domains(resp. functions). Note that pseudoconvex domains are not always Stein manifolds.

The purpose of the present paper is to show the following theorem:

Main Theorem. If $M$ admits a kähler metric with positive holomorphic bisectional curvature (see, Definition(2-7)), then pseudoconvex domains are always holomorphically convex.

For the definition of holomorphic bisectional curvature and its basic properties, see S. I. Goldberg and S. Kobayashi [2].

Since the holomorphic bisectional curvature of the Fubini-Study metric of the complex projective space $\boldsymbol{P}^{n}$ is positive and $\boldsymbol{P}^{n}$ admits no exceptional sets in the sense of $\mathrm{H}$. Grauert [5], we find that pseudoconvex domains in $\boldsymbol{P}^{n}$ are always Stein manifolds, which implies the result of

Communicated by S. Nakano, November 20, 1975.

* University of Nihon, Tokyo.

1) The author was a visiting member at the Research Institute for Mathematical Science, Kyoto University during a part of the period of preparation of this work. 
R. Fujita [1] and A. Takeuchi [8].

We shall prove our Main Theorem by using the variations of arc lengths which are well known in Riemannian Geometry (see, Lemma $(2,3)$ in $\S 2$ ) and by using a nice property of real analytic kähler metrics which is essentially due to A. Takeuchi (see, $\operatorname{Lemma}(2,10)$ in $\S 2$ ).

For a point $p \in D$, we set

$$
d(p)=\inf _{q \in \partial D} d(p, q) \quad \text { and } \quad \varphi(p)=-\log d(p) .
$$

The essential step in the proof of Main Theorem is the following Lemma (for the proof, see $\S 4$ ):

Key Lemma. Let $D$ be an s-pseudoconvex domain whose boundary is a real submanifold in $M$. Suppose that the holomorphic bisectional curvature is bounded below by a positive constant $\rho$. Then there exists a positive constant $\delta^{*}$ such that

$$
W(\varphi) \geqq \rho / 8 \text { for } p \in D_{\delta^{*}},
$$

where $W(\varphi)$ means the infimum of the eigenvalues of the Hessian of $\varphi$ on $D_{\delta^{*}}$ and $D_{\delta}=\{p \in D: d(p)<\delta\}$.

By this Lemma we prove our Main Theorem in $\S 5$.

The author understands from Professor K. Saito that Professor G. Elencwajg has announced a similar result at A. M. S. Summer Institute 1975 (Williamstown). The present work was done independently of this.

The author would like to express his hearty thanks to Professors S. Nakano, H. Komatsu, S. Iitaka, A. Takeuchi and K. Saito and Mr. H. Omoto and Mr. T. Sasaki during the preparation of the present paper.

\section{§ 2. Variations of Arc Lengths and Real Analytic Kähler Metrics}

First we consider a Riemannian manifold M. We follow notations in S. Kobayashi and K. Nomizu [7]. For vectors X, Y we denote their symetric inner product by $g(\mathrm{X}, \mathrm{Y})$. Connections, covariant differentiations and Riemannian curvature tensor are assumed to be induced from the inner product $g$ in a canonical manner. To state the theorem of Synge, 
we prepare more notations: Let $N$ and $P$ be two submanifolds in $M$ and let $p$ and $q$ be two points in $N$ and $P$ respectively. Also suppose that a geodesic curve $\sigma$ between $p$ and $q$ is given. A $C^{\infty}$-mapping $\psi$ from a rectangle $[-\varepsilon, \varepsilon] \times[\alpha, \beta]$ to $M$ is called a variation of $\sigma$ between $N$ and $P$, if the following condition is satisfied: For any fixed $\xi \in[-\varepsilon, \varepsilon]$, $\psi(\xi, t)$ gives a parametrization of an $\operatorname{arc} C_{\xi}$ between $N$ and $P$ and especially when $\xi=0, \psi(0, t)$ gives a parametrization of $\sigma$. We want to know the values of the first and the second derivatives of arc length of $C_{\xi}$ at $\xi=0$. By $D(\xi)$ we denote the arc length $\mathrm{C}_{\xi}$ :

$$
D(\xi)=\int_{\alpha}^{\beta}\|\dot{\psi}(\xi, t)\| d t,
$$

where $\|\dot{\psi}(\xi, t)\|$ is the length of the tangent vector $\dot{\psi}(\xi, t)$ of $\mathrm{C}_{\xi}$ at $(\xi, t)$. We set

$$
X=\psi_{*}\left(D_{1}\right) \text { and } Y=\psi_{*}\left(D_{2}\right) \text {, }
$$

where $D_{1}=\partial / \partial t$ and $D_{2}=\partial / \partial \xi$. Let $\{U\}$ be a local coordinate covering on some neighborhood of $\sigma$ whose local coordinates are denoted by $x^{1}$, $x^{2}, \cdots, x^{m}$. Suppose that $Y$ is expressed as

$$
Y=\sum_{i=1}^{m} Y^{i} \frac{\partial}{\partial x^{i}} \circ \psi \text { on } U \text {. }
$$

We define $\nabla_{D_{i}} \mathrm{Y}(i=1,2)$ by

$$
\begin{aligned}
& \nabla_{D_{1}} Y=\sum_{k=1}^{m} \frac{\partial Y^{k}}{\partial t} \frac{\partial}{\partial x^{k}} \circ \psi+\sum_{i, j, k=1}^{m} \frac{\partial\left(x^{i} \circ \psi\right)}{\partial t} Y^{j} \Gamma_{i j}^{k} \circ \psi \frac{\partial}{\partial x^{k}} \circ \psi, \\
& \nabla_{D_{2}} Y=\sum_{k=1}^{m} \frac{\partial Y^{k}}{\partial \xi} \frac{\partial}{\partial x^{k}} \circ \psi+\sum_{i, j, k=1}^{m} \frac{\partial\left(x^{i} \circ \psi\right)}{\partial \xi} Y^{j} \Gamma_{i j}^{k} \circ \psi \frac{\partial}{\partial x^{k}} \circ \psi,
\end{aligned}
$$

where $\Gamma_{i j}^{k}$ is defined by

$$
\nabla_{\left(\partial / \partial x^{i}\right)}\left(\frac{\partial}{\partial x^{j}}\right)=\sum \Gamma_{i j}^{k} \frac{\partial}{\partial x^{k}}
$$

Now Synge's formula is stated as follows:

Lemma (2.3). (The first and the second variations of arc lengths) Suppose that $\|\dot{\psi}(0, t)\|=1$ for each $t$. Then we obtain

(i) $D^{\prime}(0)=\left.g(X, Y) \circ \psi(0, t)\right|_{\alpha} ^{\beta}$, 
(ii) $\quad D^{\prime \prime}(0)=\int_{\alpha}^{\beta} g\left(\nabla_{D_{1}} \widetilde{Y}, \nabla_{D_{1}} \widetilde{Y}\right) \circ \psi(0, t) d t$

$$
-\int_{\alpha}^{\beta} g(R(Y, X) X, Y) \circ \psi(0, t) d t+\left.g\left(\nabla_{D_{2}} Y, X\right) \circ \psi(0, t)\right|_{\alpha} ^{\beta},
$$

where $\widetilde{Y}=Y-g(Y, X) X$.

For the proof of this Lemma, see D. Gromoll, W. Klingenberg and W. Meyer [6, Satz, p. 122].

In what follows we restrict ourselves to kähler manifolds. Let $\mathrm{M}$ be a kähler manifold whose metric is denoted by

$$
d s^{2}=2 \sum g_{\alpha \bar{\beta}} d z^{\alpha} \cdot d \bar{z}^{\beta} \text {. }
$$

As stated in Introduction, we assume that $g_{\alpha \bar{\beta}}(\alpha, \beta=1,2, \cdots, n)$ are real analytic functions. In a canonical manner, $M$ may be considered as a Riemannian manifold by the following metric:

$$
g_{\alpha \bar{\beta}}=g\left(\frac{\partial}{\partial z^{\alpha}}, \frac{\partial}{\partial \bar{z}^{\beta}}\right) .
$$

By definition, we see that

$$
g_{\alpha \beta}=g_{\bar{\alpha} \bar{\beta}}=0, \quad \overline{g_{\alpha \bar{\beta}}}=g_{\bar{\alpha} \beta} \quad \text { and } \quad g_{\alpha \bar{\beta}}=g_{\bar{\beta} \alpha} .
$$

When we say connections or Riemannian curvature tensors, we mean ones which are induced from the above metric by the canonical way. By $J$ we denote the complex structure of $M$.

We set

$$
z^{\alpha}=x^{\alpha}+\sqrt{-1} y^{\alpha}, \quad \bar{z}^{\alpha}=x^{\alpha}-\sqrt{-1} y^{\alpha}
$$

and

$$
\frac{\partial}{\partial z^{\alpha}}=\frac{1}{2}\left(\frac{\partial}{\partial x^{\alpha}}-\sqrt{-1} \frac{\partial}{\partial y^{\alpha}}\right), \quad \frac{\partial}{\partial \bar{z}^{\alpha}}=\frac{1}{2}\left(\frac{\partial}{\partial x^{\alpha}}+\sqrt{-1} \frac{\partial}{\partial y^{\alpha}}\right)
$$

We infer that

$$
J\left(\frac{\partial}{\partial x^{\alpha}}\right)=\frac{\partial}{\partial y^{\alpha}} \quad \text { and } \quad J\left(\frac{\partial}{\partial y^{\alpha}}\right)=-\frac{\partial}{\partial x^{\alpha}}
$$

In terms of holomorphic coordinates $z^{1}, z^{2}, \cdots, z^{n}$, connections and some of Riemannian curvature tensors can be expressed as follows (see, S. Kobayashi and K. Nomizu [7, II, P. 157]): 


$$
\begin{aligned}
\Gamma_{\beta \gamma}^{* \alpha} & =\sum_{\varepsilon=1}^{n} g^{\alpha \bar{\varepsilon}} \frac{\partial g_{\bar{\varepsilon} \beta}}{\partial z^{\tau}}, \\
K_{\alpha \bar{\beta} \gamma \bar{\delta}} & =\frac{\partial^{2} g_{\alpha \bar{\beta}}}{\partial z^{\tau} \partial \bar{z}^{\delta}}-\sum_{\tau, \varepsilon=1}^{n} g^{\bar{\varepsilon} \tau} \frac{\partial g_{\alpha \bar{\varepsilon}}}{\partial z^{\tau}} \cdot \frac{\partial g_{\bar{\beta} \tau}}{\partial \bar{z}^{\delta}}
\end{aligned}
$$

where $\left|g^{\alpha \bar{\beta}}\right|$ denotes the inverse matrix of $\left|g_{\alpha \bar{\beta}}\right|$. We see that

$$
K_{\alpha \bar{\beta} \gamma \bar{\delta}}=K_{\gamma \bar{\delta} \alpha \bar{\beta}}=-K_{\alpha \bar{\beta} \bar{\delta} \gamma}=-K_{\bar{\beta} \alpha \gamma \bar{\delta}}, \quad \bar{K}_{\alpha \bar{\beta} \gamma \bar{\delta}}=K_{\bar{\alpha} \beta \bar{\gamma} \delta} .
$$

Definition $(2 \cdot 7)$. (1) The metric $(2 \cdot 4)$ is said to have positive holomorphic bisectional (resp. positive sectional) curvature if there exists a positive constant $\rho$ for every point $p$ in $M$ such that the following inequality holds for every pair of vectors $X, Y \in T_{p}(M)$ :

$$
g(R(Y, X) X, Y)+g(R(Z, X) X, Z)
$$

$$
\geqq \rho\left[\left\{g(X, X) \cdot g(Y, Y)-g(X, Y)^{2}\right\}+\left\{g(X, X) \cdot g(Z, Z)-g(Z, X)^{2}\right\}\right],
$$

where $Z=J(Y)$,

(resp. $\left.g(R(Y, X) X, Y) \geqq \rho\left\{g(X, X) \cdot g(Y, Y)-g(X, Y)^{2}\right\}\right)$.

We prepare two lemmas on real analytic kähler metrics. The first lemma is a slight generalization of Lemma 2 in A. Takeuchi [9, p. 333]. By using this lemma we prove the second lemma, which will be used in the proof of Key Lemma.

Lemma (2.9). For any point $p \in M$ and for any positive constant $C_{0}$ with $C_{0}>1$, there exist a neighborhood $V(p)$ and a positive constant $\delta^{*}$ such that for any point $q \in V(p)$ and for any geodesic $\sigma$ through $q$, there exists a system of local coordinates $z^{1}, z^{2}, \cdots, z^{n}$ on $\bar{B}_{\tilde{o}^{*}}(q)$, where $B_{\delta}(q)=\{p \in M: d(p, q)<\delta\}$ and $\bar{B}_{\delta^{*}}(q)$ denotes the closure of $B_{0^{*}}(q)$, satisfying the following conditions:

(1) $\sigma$ is expressed as follows: Im $z^{1}=0, z^{2}=z^{3}=\cdots=z^{n}=0$,

(2) $2 g_{\alpha \bar{\beta}}=\delta_{\alpha \bar{\beta}}+\sum 2 K_{\alpha \bar{\beta} \gamma \delta}(0) z^{\gamma} \bar{z}^{\hat{\delta}}+\cdots$ on $\bar{B}_{\delta^{*}}(q)$,

(3) $2 g_{1, \overline{1}}=1$ on $\sigma$,

(4) $\left\|2 g_{\alpha \bar{\beta}}\right\|<C_{0}$ and $\left\|\frac{1}{2} g^{\alpha \bar{\beta}}\right\|<C_{0}$ on $\overline{B_{\delta^{*}}}(q)$,

where $\|f\|$ is defined as follows: For a real analytic function $f$ with 


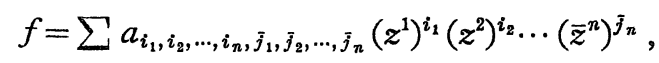

we define

$$
\|f\|=\sum\left|a_{i_{1}, i_{2}, \ldots, \bar{j}_{n}}\left(z^{1}\right)^{i_{1}}\left(z^{2}\right)^{i_{2}} \cdots\left(\bar{z}^{n}\right)^{\bar{j}_{n}}\right|
$$

The proof is almost the same as that of Lemma 2 in A. Takeuchi [9]. By this Lemma, we prove the following Lemma:

Lemma (2.10). (i) For any point $p$ and for any positive constant $C_{0}$ with $C_{0}>1$, there exist positive numbers $\delta, \delta_{0}$ and a neighborhood $V(p)$ such that for any point $q \in V(p)$ and for any geodesic $\sigma$ through $q$, there exist a local coordinate neighborhood $U(p)$ and a system of local coordinates $z^{1}, z^{2}, \cdots, z^{n}$ on $U(q)$ with following properties:

$$
B_{\delta}(q) \subset U_{\delta_{0} / 2}(z: q) \subset \bar{U}_{\delta_{0}}(z: q) \subset U(q),
$$

where $U_{\sigma}(z: q)=\left\{\left(z^{1}, z^{2}, \cdots, z^{n}\right):{ }_{1} z^{1}|<\sigma,| z^{2}|<\sigma, \cdots,| z^{n} \mid<\sigma\right\}$,

(1) $\sigma$ is expressed as Im $z^{1}=0, z^{2}=z^{3}=\cdots=z^{n}=0$,

(2) $2 g_{\alpha \bar{\beta}}=\delta_{\alpha \bar{\beta}}+\sum 2 K_{\alpha \bar{\beta} \gamma \delta}(0) z^{\gamma} \bar{z}^{\delta}+\cdots$ on $\bar{U}_{\delta_{0}}(z: q)$,

(3) $2 g_{1, \mathrm{I}}=1$ on $\sigma$,

(4) $\quad\left\|2 g_{\alpha \bar{\beta}}\right\|<C_{0}$ and $\left\|\frac{1}{2} g^{\alpha \bar{\beta}}\right\|<C_{0}$ on $\overline{U_{\bar{o}_{0}}}(z: q)$.

(ii) For any relatively compact domain $D$ in $M$ and for any positive constant $C_{0}$ with $C_{0}>1$, there exist positive constants $\delta, \delta_{0}{ }^{(1)}$, $\delta_{0}{ }^{(2)}, \cdots, \delta_{0}{ }^{(N)}$ such that for any point $p \in \bar{D}$ and for any geodesic $\sigma$ through $p$, there exist $\delta_{0}{ }^{(i)}$ and a local coordinate neighborhood $U_{\delta_{0}(i)}$ $(z: q)$ satisfying $(0) \sim(4)$ in (i) with respect to a certain system of local coordinates $z^{1}, z^{2}, \cdots, z^{n}$.

Proof of (i). We choose a system of local coordinates $w_{1}, w_{2}$, $\cdots, w_{n}$ at $p$ and by $g_{\alpha \bar{\beta}}$ we denote the metric tensor with respect to these local coordinates. By A. Takeuchi [7, p. 329] we can find a neighborhood $V(p)$ and a system of real analytic functions $\lambda_{\gamma \delta}(w)(\gamma, \delta$ $=1,2, \cdots, n)$ on $V(p)$ such that

$$
\sum_{\alpha, \beta=1}^{n} g_{\alpha \bar{\beta}} \lambda_{\alpha r} \overline{\lambda_{\beta \delta}}=\delta_{\tau \delta} \quad \text { on } \quad V(p)
$$


Take an arbitrary point $q$ in $V(p)$. We set $w_{i}(q)=\zeta_{i}$ for $i=1,2, \cdots, n$. We define a system of local coordinates $z_{1}{ }^{\prime}, z_{2}{ }^{\prime}, \cdots, z_{n}{ }^{\prime}$ by

$$
w_{\alpha}-\zeta_{\alpha}=\sum_{\beta=1}^{n} \lambda_{\alpha \beta} z_{\beta}^{\prime} \quad \text { for } \alpha=1,2, \cdots, n \text {. }
$$

Now setting

$$
u_{\alpha}=z_{\alpha}^{\prime}+\sum_{k=2}^{\infty} \sum_{i_{1}+i_{2}+\cdots+i_{n}=k} \beta_{i_{1}, i_{2}, \cdots, i_{n}}^{(\alpha)} z_{1}{ }^{\prime i_{1}}{z_{2}}^{\prime i_{2}} \cdots z_{n}{ }^{\prime i_{n}} . \quad(\alpha=1,2, \cdots, n),
$$

we can determine $\left\{\beta_{i_{1}, i_{2}, \cdots, i_{n}}^{(\alpha)}\right\}$ uniquely so that they satisfy the condition (2). Moreover, after $\mathrm{A}$. Takeuchi, we may assume that $u_{1}, u_{2}, \cdots, u_{n}$ give local coordinates on $\bar{B}_{\delta^{*}}(q)$ (see, Lemma 1 in A. Takeuchi [9]).

Now we choose a constant $\delta_{0}^{\prime}$ so that $U_{\delta_{0^{\prime}}}(u: q) \subset B_{\delta^{*}}(q)$. We denote the supremum of such $\delta_{0}^{\prime}$ by $\delta_{0}^{\prime}(\mathrm{q})$. Then letting $\delta_{0}{ }^{\prime \prime}=\inf _{q \in \boldsymbol{V}(p)} \delta_{0}{ }^{\prime}(q)$, we see that $\delta_{0}^{\prime \prime}>0$. Next we choose an arbitrary geodesic $\sigma$ through $q$. We choose a unitary matrix $\left|\alpha_{i, j}\right|$ and a system of local coordinates $u_{1}^{\prime}$, $u_{2}{ }^{\prime}, \cdots, u_{n}{ }^{\prime}$ with

$$
u_{i}^{\prime}=\sum_{j=1}^{n} \alpha_{i, j} u_{j} \quad(i=1,2, \cdots, n)
$$

such that the tangent vector of $\sigma$ at $u_{1}{ }^{\prime}=0, u_{2}{ }^{\prime}=0, \cdots, u_{n}{ }^{\prime}=0$ is normalized as follows:

$$
\frac{d u_{1}^{\prime}}{d s}(0)=1 \quad \text { and } \quad \frac{d u_{i}^{\prime}}{d s}(0)=0 \quad(i=2, \cdots, n)
$$

where $s$ denotes the length of $\sigma$. We infer that $U_{\hat{\delta}_{0} / n}\left(u^{\prime}: q\right) \subset \bar{B}_{\hat{o}^{*}}(q)$ Owing to the real analyticity of the metric tensor, $\sigma$ can be expressed as

$$
u_{i}^{\prime}=\sum_{k=1}^{\infty} a_{i, k} s^{k} \quad(i=1,2, \cdots, n)
$$

Replacing $s$ by $z^{1}=s+\sqrt{-1} t$ in $u_{1}^{\prime}$ and using the implicit function theorem, we obtain

$$
z^{1}=\varphi\left(u_{1}^{\prime}\right)
$$

Set

$$
z^{1}=\varphi\left(u_{1}^{\prime}\right), \quad z^{2}=u_{2}^{\prime}, \cdots, z^{n}=u_{n}^{\prime} .
$$

Then we get again a new system of local coordinates on $\bar{B}_{\sigma^{*}}(q)$ satisfying 
(1) (3) (see, section 5 in A. Takeuchi [9]). With referrence to the univalency of $(2 \cdot 11)$ and $d u_{1}{ }^{\prime} / d z^{1}(0)=1$, we see that $U_{\delta_{0}}(z: q) \subset \bar{B}_{\delta^{*}}(q)$, where $\delta_{0}=\delta_{0}{ }^{\prime \prime} / 4 n$. Then there exists a constant $\underline{\delta}$ such that $B_{\underline{\delta}}(q) \subset U_{\delta_{0} / 2}$ $(z: q)$. We denote the supremum of such $\underline{\delta}$ by $\delta(q)$. Then we see that $\delta=\inf _{q \in \boldsymbol{V}(p)} \delta(q)$ is positive. So we see that $z^{1}, z^{2}, \cdots, z^{n}$ satisfy $(0) \sim(3)$. Finally making $V(p)$ smaller, we can staisfy $(0) \sim(4)$. This completes the proof.

Proof of (ii). For each $p \in \bar{D}$ we choose $V(p)$ and $\delta(p), \delta_{0}(p)$ by using (i). We can cover $\bar{D}$ by $\left\{V\left(p_{i}\right)\right\} \quad(i=1,2, \cdots, N)$. Letting $\delta_{0}{ }^{(i)}$ $=\delta_{0}\left(p_{i}\right) \quad(i=1,2, \cdots, N)$ and $\delta=\min \delta\left(p_{i}\right)$, we see that $\delta, \delta_{0}{ }^{(1)}, \delta_{0}{ }^{(2)}, \cdots, \delta_{0}{ }^{(1)}$ satisfy the condition in (ii).

In terms of the real coordinates $x^{1}, y^{1}, \cdots, x^{n}, y^{n}$, we denote connection coefficient $\Gamma_{j, k}^{i}$ by

$$
\begin{aligned}
& \nabla_{\left(\partial / \partial x^{i}\right)}\left(\frac{\partial}{\partial x^{j}}\right)=\sum_{k=1}^{n} \Gamma_{i, j}^{k} \frac{\partial}{\partial x^{k}}+\sum_{k=1}^{n} \Gamma_{i, j}^{n+k} \frac{\partial}{\partial y^{k}}, \\
& \nabla_{\left(\partial / \partial x^{i}\right)}\left(\frac{\partial}{\partial y^{j}}\right)=\sum_{k=1}^{n} \Gamma_{i, n+j}^{k} \frac{\partial}{\partial x^{k}}+\sum_{k=1}^{n} \Gamma_{i, n+j}^{n+k} \frac{\partial}{\partial y^{k}}, \\
& \nabla_{\left(\partial / \partial y^{i}\right)}\left(\frac{\partial}{\partial x^{j}}\right)=\sum_{k=1}^{n} \Gamma_{n+i, j}^{k} \frac{\partial}{\partial x^{k}}+\sum_{k=1}^{n} \Gamma_{n+i, j}^{n+k} \frac{\partial}{\partial y^{k}}, \\
& \nabla_{\left(\partial / \partial y^{i}\right)}\left(\frac{\partial}{\partial y^{j}}\right)=\sum_{k=1}^{n} \Gamma_{n+i, n+j}^{k} \frac{\partial}{\partial x^{k}}+\sum_{k=1}^{n} \Gamma_{n+i, n+j}^{n+k} \frac{\partial}{\partial y^{k}} .
\end{aligned}
$$

Because the connection is induced by the metric, we see that $\Gamma_{j, i}^{k}=\Gamma_{i, j}^{k}$ for $i, j, k=1,2, \cdots, 2 n$. Also we have

$$
\nabla_{\left(\partial / \partial x^{i}\right)}\left(\frac{\partial}{\partial y^{j}}\right)=J\left(\nabla_{\left(\partial / \partial x^{i}\right)}\left(\frac{\partial}{\partial x^{j}}\right)\right)
$$

and

$$
\nabla_{\left(\partial / \partial x^{i}\right)}\left(\frac{\partial}{\partial x^{j}}\right)=\nabla_{\left(\partial / \partial x^{j}\right)}\left(\frac{\partial}{\partial x^{i}}\right), \quad \nabla_{\left(\partial / \partial x^{i}\right)}\left(\frac{\partial}{\partial y^{j}}\right)=\nabla_{(\partial / \partial y) i}\left(\frac{\partial}{\partial x^{i}}\right) .
$$

By these relations, we obtain

$$
\left\{\begin{array}{l}
\Gamma_{i, n+j}^{k}=-\Gamma_{i, j}^{n+k}, \quad \Gamma_{n+i, n+j}^{k}=-\Gamma_{n+i, j}^{n+k}, \quad \Gamma_{n+i, n+j}^{k}=-\Gamma_{i, j}^{k} \\
\Gamma_{i, n+j}^{n+k}=\Gamma_{i, j}^{k}, \quad \Gamma_{n+i, n+j}^{n+k}=\Gamma_{n+i, j}^{k}, \quad \Gamma_{n+i, n+j}^{n+k}=-\Gamma_{i, j}^{n+k}
\end{array}\right.
$$

Recall that $\sigma$ is a geodesic. Then with reference to (1) in (i), 
Lemma $(2 \cdot 10)$, we see that

$$
\Gamma_{1, \overline{1}}^{j}=0 \text { and } \Gamma_{1, n+1}^{j}=0 \text { on } \sigma(j=1,2, \cdots, 2 n),
$$

and that

$$
g\left(\frac{\partial}{\partial x^{1}}, \frac{\partial}{\partial x^{1}}\right)=1 \text { and } g\left(\frac{\partial}{\partial x^{1}}, \frac{\partial}{\partial y^{1}}\right)=0 \text { on } \sigma
$$

We prepare two propositions which will be used in the proof of Key Lemma (see, § 4):

Proposition (2-15). Let $D$ be a relatively compact domain and let $C_{0}$ be a positive constant with $C_{0}>1$. Suppose that for a point $p \in \bar{D}$, a geodesic $\sigma$ through $p$ is given. We choose local coordinates as in (ii), Lemma (2.10). Moreover, suppose that $\phi: \boldsymbol{R} \rightarrow M$ is a mapping defined by $z^{1}=t, z^{2}=0, \cdots, z^{n}=0$. Then we have

$$
\begin{aligned}
& g\left(\frac{\partial}{\partial x^{j}}, \frac{\partial}{\partial x^{i}}\right) \circ \phi=\delta_{i, j}+2 \operatorname{Re} K_{i \bar{j} \overline{1} \overline{1}}(0) \cdot t^{2}+O\left(t^{3}\right), \\
& g\left(\frac{\partial}{\partial y^{j}}, \frac{\partial}{\partial x^{i}}\right) \circ \phi=2 \operatorname{Im} K_{i \bar{j} \overline{1}}(0) \cdot t^{2}+O\left(t^{3}\right), \\
& \Gamma_{i, j}^{k} \circ \phi=2 \operatorname{Re} K_{\dot{k} i \overline{1} j}(0) \cdot t+O\left(t^{2}\right), \\
& \Gamma_{i, j}^{n+k} \circ \phi=2 \operatorname{Im} K_{\bar{k} i \overline{1} j}(0) \cdot t+O\left(t^{2}\right),
\end{aligned}
$$

(4) by $(2 \cdot 13)$

$$
\operatorname{Re} K_{j \overline{1} \overline{1}}(0)=0 \text { and } \operatorname{Im} K_{j \overline{1} 1 \overline{1}}(0)=0 .
$$

These follow from

$$
\begin{aligned}
& g\left(\frac{\partial}{\partial x^{i}}, \frac{\partial}{\partial x^{j}}\right)=\left\{g\left(\frac{\partial}{\partial z^{i}}, \frac{\partial}{\partial \bar{z}^{j}}\right)+g\left(\frac{\partial}{\partial z^{i}}, \frac{\partial}{\partial \bar{z}^{j}}\right)\right\}, \\
& g\left(\frac{\partial}{\partial x^{i}}, \frac{\partial}{\partial y^{j}}\right)=\frac{1}{\sqrt{-1}}\left\{g\left(\frac{\partial}{\partial z^{i}}, \frac{\partial}{\partial \bar{z}^{j}}\right)-g\left(\frac{\partial}{\partial z^{i}}, \frac{\partial}{\partial \bar{z}^{j}}\right)\right\},
\end{aligned}
$$

and

$$
\nabla_{\left(\partial / \partial x^{i}\right)}\left(\frac{\partial}{\partial x^{j}}\right)=\sum_{k=1}^{n} \operatorname{Re} \Gamma_{i, j}^{* k} \frac{\partial}{\partial x^{k}}+\sum_{k=1}^{n} \operatorname{Im} \Gamma_{i, j}^{* k} \frac{\partial}{\partial y^{k}}
$$

The terms $O\left(t^{3}\right)$ and $O\left(t^{2}\right)$ can be estimated in the following 
Proposition (2.16). Let $\phi$ be the mapping given in Proposition $(2 \cdot 15)$ and let $\delta_{0}=\min _{i=1,2, \ldots, N} \delta_{0}{ }^{(i)}$. Then we have

(1) Suppose that $g\left(\partial / \partial x^{i}, \partial / \partial x^{j}\right) \circ \phi=t^{m} \cdot G(t)$, where $G(t)$ is a real analytic function. Then we obtain

$$
|G(t)| \leqq 2 C_{0} \delta_{0}^{-m} \text { for } t \text { with } \phi(t) \in B_{\delta}(p),
$$

(2) Suppose that $g\left(\partial / \partial x^{i}, \partial / \partial y^{j}\right) \circ \phi=t^{m} \cdot G^{\prime}(t)$, where $G^{\prime}(t)$ is a real analytic function. Then we obtain

$$
\left|G^{\prime}(t)\right| \leqq 2 C_{0} \delta_{0}^{-m} \text { for } t \text { with } \phi(t) \in B_{\delta}(p),
$$

(3) Suppose that $\partial / \partial t\left(g \partial / \partial x^{i}, \partial / \partial y^{j}\right) \circ \phi=t^{m} \cdot G^{\prime \prime}(t)$, where $G^{\prime \prime}(t)$ is a real analytic function. Then we obtain

$$
\left|G^{\prime \prime}(t)\right| \leqq C(m) C_{0} \delta_{0}^{-(m+1)} \text { for } t \text { with } \phi(t) \in B_{\delta}(p),
$$

(4) Suppose that $\Gamma_{i, j}^{k} \circ \phi=t^{m} \cdot G^{\prime \prime \prime}(t)$, where $G^{\prime \prime \prime}(t)$ is a real analytic function. Then we obtain

$$
\left|G^{\prime \prime \prime}(t)\right| \leqq n C(m) C_{0}^{2} \delta_{0}^{-(m+1)} \text { for } t \text { with } \phi(t) \in B_{\delta}(t),
$$

where $C(m)=4^{m+2} \cdot 3^{-(m-1)}$.

Let

$$
2 g_{\alpha \bar{\beta}}=\sum a_{i_{1} \cdots i_{n}, \tilde{j}_{1} \cdots \bar{j}_{n}}\left(z^{1}\right)^{i_{1} \cdots\left(\bar{z}^{n}\right)^{\bar{j}_{n}}} .
$$

Then owing to (4) in Lemma $(2 \cdot 10)$, we find

$$
\left|a_{i_{1} \cdots \bar{j}_{n}}\left(z^{1}\right)^{i_{1}} \cdots\left(\bar{z}^{n}\right)^{\bar{j}_{n}}\right| \leqq C_{0}\left|\left(z^{1} / \delta_{0}{ }^{(i)}\right)^{i_{1}} \cdots\left(\bar{z}^{n} / \delta_{0}{ }^{(i)}\right)^{\bar{j}_{n}}\right| \text { on } U_{\delta_{0}(i)}(z: p) .
$$

By using (0) in (ii) in Lemma $(2 \cdot 10)$ and the definition of $\delta_{0}$, we obtain the above estimates.

\section{§ 3. Some Propositions Concerning Pseudoconvexity}

In this section we summarize some propositions concerning pseudoconvex functions and pseudoconvex domains. Let $z^{1}, z^{2}, \cdots, z^{n}$ be a system of local coordinates on some neighborhood of $p$ and let $L$ be a complex line through $p$ which is parametrized as

$$
L: z^{1}=a_{1} \lambda, z^{2}=a_{2} \lambda, \cdots, z^{n}=a_{n} \lambda,
$$

where

$$
\left|a_{1}\right|^{2}+\left|a_{2}\right|^{2}+\cdots+\left|a_{n}\right|^{2}=1 \text {. }
$$


A function $\varphi$ is called a pseudoconvex function on $D$, if for any point $p \in D$ and for any complex line $L$,

$$
\varphi(0) \leqq \frac{1}{2 \pi} \int_{0}^{2 \pi} \varphi\left(r e^{i \theta}\right) d \theta \quad \text { where } \quad \lambda=r e^{i \theta}
$$

In what follows we write

$$
\mu_{r, p}(\varphi)=\frac{1}{2 \pi} \int_{0}^{2 \pi} \varphi\left(r e^{i \theta}\right) d \theta
$$

When $\varphi$ is a function of $C^{2}$-class, we find that

$$
\Delta \varphi(0)=4 \lim _{r \rightarrow 0} \frac{\mu_{r, p}(\varphi)-\varphi(0)}{r^{2}}
$$

where

$$
\Delta \varphi=4 \frac{\partial^{2} \varphi}{\partial \lambda \partial \bar{\lambda}}
$$

Hence if $\varphi$ is a pseudoconvex function of $C^{2}$-class, we see that $\Delta \varphi \geqq 0$ holds everywhere. More generally a necessary and sufficient condition that $\varphi$ is a pseudoconvex (resp. $s$-pseudoconvex) function is that

$$
W(\varphi(p))=4 \inf _{L} \lim _{r \rightarrow 0} \frac{\inf _{r, p}(\varphi)-\varphi(0)}{r^{2}}
$$

is non-negative (resp. positive) at every point $p$ in $D$.

The following theorem is well known (for example, see H. Grauert [4]):

Theorem (3.2). If $D$ is a relatively compact s-pseudoconvex domain, then $D$ is holomorphically convex.

Let $D$ be an $s$-pseudoconvex domain whose boundary is a real submanifold in $M$. Take a point $p \in D$ near the boundary. Then we may assume that there exist a boundary point $q$ and a geodesic $\sigma$ between $p$ and $q$ which attains $d(p)=d(p, q)$. So there exist a local coordinate neighborhood $U_{\delta_{0}}$ of $p$ and a system of local coordinates $z^{1}, z^{2}, \cdots, z^{n}$ which satisfies $(0) \sim(4)$ in (i) in Lemma $(2 \cdot 10)$.

Then we can prove the following Proposition, which will be used in $\S 4$. For the proof, see A. Takeuchi [9, Lemma 3, p. 338]: 
Proposition (3.3). There exist a holomorphic function $\beta\left(z^{2}, z^{3}, \cdots\right.$, $z^{n}$ ) on $U_{\hat{o} 0}$ and a divisor $S$ through $q$,

$$
S:\left\{z^{1}=\beta\left(z^{2}, z^{3}, \cdots, z^{n}\right)\right\}
$$

such that

$$
S \cap D=\phi \text { and } S \cap \partial D=\{q\} .
$$

Now we consider a general pseudoconvex domain $D$. Let $p_{0}$ be a boundary point and let $U\left(p_{0}\right)$ be a Stein neighborhood. Then $D \cap U\left(p_{0}\right)$ is also a Stein manifold. Hence there exists a sequence of domains in $D \cap U\left(p_{0}\right), \Delta_{j},(j=1,2, \cdots)$ with the following property: (1) $\Delta_{j} \subset \Delta_{j+1}, \cup_{j=1}^{\infty} \Delta_{j}$ $=U\left(p_{0}\right) \cap D$ and (2) $\Delta_{j}$ is an $s$-pseudoconvex domain whose boundary is a real submanifold in $D \cap U\left(p_{0}\right)$.

We infer the following Proposition:

Proposition (3.4). For any point $p_{0} \in \partial D$ and for any neighborhood $U\left(p_{0}\right)$ there exists a relatively compact neighborhood $V\left(p_{0}\right)$ in $U\left(p_{0}\right)$ satisfying

$$
d(p)=\inf _{q \in\left(\partial \boldsymbol{D} \cap U\left(p_{0}\right)\right)} d(p, q) \quad \text { for } p \in V\left(p_{0}\right)
$$

For the proof, see H. Grauert [3, Hilfsatz 9, p. 69].

Take an arbitrary point $p$ in $V\left(p_{0}\right)$. Then there exists an integer $i_{0}$ such that $p \in \Delta_{i}$ for $i \geqq i_{0}$. We set

$$
d_{i}(p)=\inf _{q \in \partial \Delta_{j}} d(p, q) \text { and } \varphi_{i}(p)=-\log d_{i}(p) \text { for } p \in \Delta_{j} .
$$

Then we see that

$$
d_{i}(p)<d_{i+1}(p) \text { and } d_{j}(p) \rightarrow d(p) \quad(j \rightarrow \infty),
$$

and that

$$
\varphi_{i}(p)>\varphi_{i+1}(p) \text { and } \varphi_{j}(p) \rightarrow \varphi(p) \quad(j \rightarrow \infty) .
$$

We note that if $\varphi_{i}$ is a pseudoconvex function (resp. $W\left(\varphi_{i}\right) \geqq c>0$ ) for $i \geqq i_{0}$, then $\varphi$ is also a pseudoconvex function (resp. $\left.W(\varphi) \geqq c\right)$.

\section{§4. Proof of Key Lemma}

We shall prove our Key Lemma stated in Introduction. Let $\mathrm{D}$ be 
an $s$-pseudoconvex domain whose boundary is a real submanifold in $M$. Take a positive constant $C_{0}$ with $C_{0}>1$. By (ii) in Lemma $(2 \cdot 10)$, we can find positive constants $\delta, \delta_{0}{ }^{(1)}, \delta_{0}{ }^{(2)}, \cdots, \delta_{0}{ }^{(N)}$. As in Proposition $(2 \cdot 16)$, we define $\delta_{0}$. Choosing $\delta^{\prime}\left(\delta^{\prime}<\delta\right)$ very small, we may assume that the following condition is satisfied: For any point $p \in D_{\delta^{\prime}}$, there exist a point $q \in \partial D$ and a geodesic $\sigma$ between $p$ and $q$ satisfying $d(p)$ $=d(p, q)$.

Now we take a point $p$ in $D_{\hat{\delta}^{\prime}}$. Then we can find $q$ and $\sigma$ as above. By (ii) in Lemma $(2 \cdot 10)$, we choose a system of local coordinates $z^{1}, z^{2}, \cdots$, $z^{n}$. Letting $d(p)=l$, we see that $l<\delta$. To get the estimate $W(\varphi)$ on $D_{\delta^{\prime}}$, we construct another simple variation of $\sigma$. Let $N$ be a complex line through $p$ and its holomorphic parametrization is given as follows:

$$
N: z^{1}=a_{1} \lambda, z^{2}=a_{2} \lambda, \cdots, z^{n}=a_{n} \lambda,
$$

where

$$
\left|a_{1}\right|^{2}+\left|a_{2}\right|^{2}+\cdots+\left|a_{n}\right|^{2}=1 \text {. }
$$

We write $\lambda=\xi+\sqrt{-1} \eta$.

Choose a divisor $S:\left\{z^{1}=\beta\left(z^{2}, z^{3}, \cdots, z^{n}\right)\right\}$ through $q$ as in Proposition $(3 \cdot 3)$. By the choice of the coordinates, we find $z^{1}(q)=l$ and

$$
l=\operatorname{Re} \beta(0,0, \cdots, 0) \text { and } 0=\operatorname{Im} \beta(0,0, \cdots, 0) .
$$

Consider a holomorphic variation $\psi$ of $\sigma$ between $\mathrm{N}$ and $\mathrm{S}, \psi: \boldsymbol{D}$ $\times[0, l] \rightarrow \mathrm{M}$ as follows, where $\boldsymbol{D}$ is a small disk:

$$
\left\{\begin{array}{l}
z^{1}=\left(1-\frac{t}{l}\right) a_{1} \lambda+\frac{t}{l} \beta\left(a_{2} \lambda, a_{3} \lambda, \cdots, a_{n} \lambda\right) \\
z^{2}=a_{2} \lambda \\
\vdots \\
z^{n}=a_{n} \lambda,
\end{array}\right.
$$

where we say a holomorphic variation $\psi$ of $\sigma$ if $\psi$ is a holomorphic mapping for every fixed $t$. Then the real representation of $\psi(\xi, \eta, t)$ is given as follows:

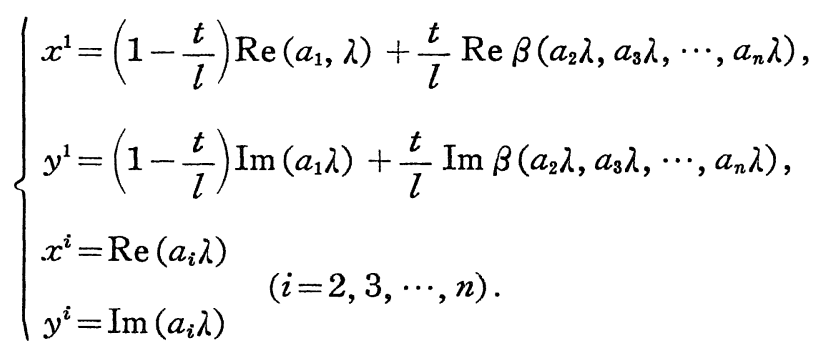


First we show

$$
\| \psi, 0,0, t) \|=1
$$

In fact, by $(4 \cdot 2)$ and $(4 \cdot 4)$ we see that $\dot{\psi}(0,0, t)=\partial / \partial x^{1} \circ \psi(0,0, t)$. And by $(2 \cdot 13)$, we prove the assertion.

Next we set

$$
X=\psi_{*}\left(D_{1}\right), Y=\psi_{*}\left(D_{2}\right) \text { and } Z=\psi_{*}\left(D_{3}\right) \text {, }
$$

where $D_{1}=\partial / \partial t, D_{2}=\partial / \partial \xi$ and $D_{3}=\partial / \partial \eta$. Then $X, Y$ and $Z$ can be expressed as follows:

$$
\begin{aligned}
X & =\left\{-\frac{1}{l} \operatorname{Re}\left(a_{1} \lambda\right)+\frac{1}{l} \operatorname{Re} \beta\right\} \frac{\partial}{\partial x^{1}} \\
& +\left\{-\frac{1}{l} \operatorname{Im}\left(a_{1} \lambda\right)+\frac{1}{l} \operatorname{Im} \beta\right\} \frac{\partial}{\partial y^{1}},
\end{aligned}
$$

$$
\begin{aligned}
Y= & \left\{\left(1-\frac{t}{l}\right) \operatorname{Re} a_{1}+\frac{t}{l} \frac{\partial \operatorname{Re} \beta}{\partial \xi}\right\} \frac{\partial}{\partial x^{1}}+\left\{\left(1-\frac{t}{l}\right) \operatorname{Im} a_{1}\right. \\
& \left.+\frac{t}{l} \frac{\partial \operatorname{Im} \beta}{\partial \xi}\right\} \frac{\partial}{\partial y^{1}}+\sum_{i=2}^{n} \operatorname{Re} a_{i} \frac{\partial}{\partial x^{i}}+\sum_{i=2}^{n} \operatorname{Im} a_{i} \frac{\partial}{\partial y^{i}}, \\
Z= & \left\{\left(1-\frac{t}{l}\right)\left(-\operatorname{Im} a_{1}\right)+\frac{t}{l} \frac{\partial \operatorname{Re} \beta}{\partial \eta}\right\} \frac{\partial}{\partial x^{1}}+\left\{\left(1-\frac{t}{l}\right) \operatorname{Re} a_{1}\right. \\
& \left.+\frac{t}{l} \frac{\partial \operatorname{Im} \beta}{\partial \eta}\right\} \frac{\partial}{\partial y^{1}}+\sum_{i=2}^{n}\left(-\operatorname{Im} a_{i}\right) \frac{\partial}{\partial x^{i}}+\sum_{i=2}^{n} \operatorname{Re} a_{i} \frac{\partial}{\partial y^{i}} .
\end{aligned}
$$

Because $\psi$ is a holomorphic variation, we find that

$$
Z=J(Y) \text {. }
$$

Now we set

$$
D^{*}(\xi, \eta)=\int_{0}^{l}\|\dot{\psi}(\xi, \eta, t)\| d t \quad \text { and } \quad \Psi(\xi, \eta)=-\log D^{*}(\xi, \eta) .
$$

We shall calulate

$$
\Delta \Psi(0,0)=\left(\frac{\partial^{2} \Psi}{\partial \xi^{2}}+\frac{\partial^{2} \Psi}{\partial \eta^{2}}\right)(0,0)
$$

For this we first calculate $\partial^{2} \Psi / \partial \xi^{2}(0,0)$ by using

$$
\frac{\partial^{2} \Psi}{\partial \xi^{2}}(0,0)=\frac{1}{\left(D^{*}\right)^{2}}\left(\frac{\partial D^{*}}{\partial \xi}\right)^{2}(0,0)-\frac{1}{D^{*}}\left(\frac{\partial^{2} D^{*}}{\partial \xi^{2}}\right)(0,0) .
$$

Referring to $(4 \cdot 5)$, we can compute $\partial D^{*} / \partial \xi(0,0)$ by Lemma $(2 \cdot 3)$ and we obtain 


$$
\frac{\partial D^{*}}{\partial \xi}(0,0)=\left.g(Y, X) \circ \psi(0,0, t)\right|_{0} ^{l} .
$$

Putting $X$ and $Y$ into $g(X, Y)$ by $(4 \cdot 6)$ and (4.7) and using (2.14), we get

$$
\begin{aligned}
g(X, Y) \circ \psi(0,0, t)=\left(1-\frac{t}{l}\right) \operatorname{Re} a_{1}+\frac{t}{l} \frac{\partial \operatorname{Re} \beta}{\partial \xi}(0) \\
\quad+\sum_{i=2}^{n} \operatorname{Re} a_{i} g\left(\frac{\partial}{\partial x^{i}}, \frac{\partial}{\partial x^{1}}\right) \circ \psi(0,0, t) \\
\quad+\sum_{i=2}^{n} \operatorname{Im} a_{i} g\left(\frac{\partial}{\partial y^{i}}, \frac{\partial}{\partial x^{1}}\right) \circ \psi(0,0, t) .
\end{aligned}
$$

By (1), (2) in Proposition (2.15) and $(2 \cdot 6)$, we see that

$$
\begin{aligned}
& g\left(\frac{\partial}{\partial x^{i}}, \frac{\partial}{\partial x^{1}}\right) \circ \psi(0,0, t)=2 \operatorname{Re} K_{i \overline{1} \overline{1}}(0) \cdot t^{2}+O\left(t^{3}\right) \quad(i=2,3, \cdots, n), \\
& g\left(\frac{\partial}{\partial y^{i}}, \frac{\partial}{\partial x^{1}}\right) \circ \psi(0,0, t)=-2 \operatorname{Im} K_{i \overline{1} 1 \overline{1}}(0) \cdot t^{2}+O\left(t^{3}\right) \quad(i=2,3, \cdots, n) .
\end{aligned}
$$

Moreover, by (4) in the same Proposition, we find $K_{i \overline{1} 1}(0)=0$. Therefore we get

$$
\begin{gathered}
g\left(\frac{\partial}{\partial y^{i}}, \frac{\partial}{\partial x^{1}}\right) \circ \psi(0,0, t)=t^{3} \cdot G_{i} \text { and } g\left(\frac{\partial}{\partial y^{i}}, \frac{\partial}{\partial x^{1}}\right) \circ \psi(0,0, t)=t^{3} \cdot G_{n+i} \\
\text { for } i=2,3, \cdots, n .
\end{gathered}
$$

Hence by (1), (2) in Proposition $(2 \cdot 16)$, we have

$$
\left|G_{i}\right| \leqq 2 C_{0} \delta_{0}^{-3} \text { and }\left|G_{n+i}\right| \leqq 2 C_{0} \delta_{0}^{-3}(\mathrm{i}=2,3, \cdots, n) .
$$

So we obtain

$$
\frac{\partial D^{*}}{\partial \xi}(0,0)=\left(\frac{\partial \operatorname{Re} \beta}{\partial \xi}(0,0)-\operatorname{Re} a_{1}\right)+M^{(1)}(l) \cdot l^{3},
$$

where

$$
\left|M^{(1)}(l)\right| \leqq 4(n-1) C_{0} \delta_{0}^{-3}
$$

Also $\partial^{2} D^{*} / \partial \xi^{2}(0,0)$ can be computed by (ii) in Lemma $(2 \cdot 3)$ as follows:

$(4 \cdot 10) \quad \frac{\partial^{2} D^{*}}{\partial \xi^{2}}(0,0)=\int_{0}^{l}\left\{g\left(\nabla_{D_{1}} \widetilde{Y}, \nabla_{D_{1}} \widetilde{Y}\right)-g(R(Y, X) X, Y)\right\} \circ \psi(0,0, t) d t$ 


$$
+\left.g\left(\nabla_{D_{2}} Y, X\right) \circ \psi(0,0, t)\right|_{0} ^{l} .
$$

The first term in the left side can be computed as follows: From $(2 \cdot 1)$ we infer that

$$
\left(\nabla_{D_{1}} \widetilde{Y}\right) \circ \psi(0,0, t)=\nabla_{D_{1}}\{\widetilde{Y} \circ \psi(0,0, t)\} .
$$

By using $X \circ \psi(0,0, t)=\partial / \partial x^{1} \circ \psi(0,0, t)$, we see that

$$
\begin{aligned}
\widetilde{Y} \circ \psi(0,0, t)= & -\left\{\sum_{i=2}^{n} \operatorname{Re} a_{i} g\left(\frac{\partial}{\partial x^{i}}, \frac{\partial}{\partial x^{1}}\right) \circ \psi(0,0, t)\right. \\
& \left.+\sum_{i=2}^{n} \operatorname{Im} a_{i} g\left(\frac{\partial}{\partial y^{i}}, \frac{\partial}{\partial x^{1}}\right) \circ \psi(0,0, t)\right\} \frac{\partial}{\partial x^{1}} \circ \psi(0,0, t) \\
& +\left\{\left(1-\frac{t}{l}\right) \operatorname{Im} a_{1}+\frac{t}{l} \frac{\partial \operatorname{Im} \beta}{\partial \xi}\right\} \frac{\partial}{\partial y^{1}} \circ \psi(0,0, t) \\
& +\sum_{i=2}^{n} \operatorname{Re} a_{i} \frac{\partial}{\partial x^{i}} \circ \psi(0,0, t)+\sum_{i=2}^{n} \operatorname{Im} a_{i} \frac{\partial}{\partial y^{i}} \circ \psi(0,0, t) .
\end{aligned}
$$

Also by $(4 \cdot 4)$ and $(2 \cdot 14)$, we have

$$
\begin{gathered}
\frac{\partial}{\partial t}\left(x^{i} \circ \psi(0,0, t)\right)=\delta_{i, 1} \text { and } \frac{\partial}{\partial t}\left(y^{i} \circ \psi(0,0, t)\right)=0 \\
(i=1,2, \cdots, n), \\
\Gamma_{1,1}^{j} \circ \psi(0,0, t)=\Gamma_{1, n+1}^{j} \circ \psi(0,0, t)=0 \quad(j=1,2, \cdots, 2 n) .
\end{gathered}
$$

Hence defining $U^{i}$ and $V^{i}(i=1,2, \cdots, n)$ by

$$
\left(\nabla_{D_{1}} \widetilde{Y}\right) \circ \psi(0,0, t)=\sum_{i=1}^{n} U^{i} \frac{\partial}{\partial x^{i}} \circ \psi(0,0, t)+\sum_{i=1}^{n} V^{i} \frac{\partial}{\partial y^{i}} \circ \psi(0,0, t),
$$

we obtain

$$
\begin{aligned}
& U^{1}=-\sum_{i=2}^{n} \operatorname{Re} a_{i} \frac{\partial}{\partial t}\left\{g\left(\frac{\partial}{\partial x^{i}}, \frac{\partial}{\partial x^{1}}\right) \circ \psi(0,0, t)\right\} \\
&-\sum_{i=2}^{n} \operatorname{Im} a_{i} \frac{\partial}{\partial t}\left\{g\left(\frac{\partial}{\partial y^{i}}, \frac{\partial}{\partial x^{1}}\right) \circ \psi(0,0, t)\right\} \\
&+\sum_{i=2}^{n} \operatorname{Re} a_{i} \Gamma_{1, i}^{1} \circ \psi(0,0, t)+\sum_{i=2}^{n} \operatorname{Im} a_{i} \Gamma_{1, n+i}^{1} \circ \psi(0,0, t) \\
& U^{k}= \sum_{i=2}^{n} \operatorname{Re} a_{i} \Gamma_{1, i}^{k} \circ \psi(0,0, t)+\sum_{i=2}^{n} \operatorname{Im} a_{i} \Gamma_{1, n+i}^{k} \circ \psi(0,0, t) \\
&(k=2,3, \cdots, n),
\end{aligned}
$$




$$
\begin{aligned}
& V^{1}=\left\{-\frac{1}{l} \operatorname{Im} a_{1}+\frac{1}{l} \frac{\partial \operatorname{Im} \beta}{\partial \xi}\right\}+\sum_{i=2}^{n} \operatorname{Re} a_{i} \Gamma_{1, i}^{n+1} \circ \psi(0,0, t) \\
&+\sum_{i=2}^{n} \operatorname{Im} a_{i} \Gamma_{1, n+i}^{n+1} \circ \psi(0,0, t), \\
& V^{k}=\sum_{i=2}^{n} \operatorname{Re} a_{i} \Gamma_{1, i}^{n+k} \circ \psi(0,0, t)+\sum_{i=2}^{n} \operatorname{Im} a_{i} \Gamma_{1, n+1}^{n+k} \circ \psi(0,0, t) \\
&(k=2,3, \cdots, n) .
\end{aligned}
$$

By (3) in Proposition $(2 \cdot 15)$ and $(2 \cdot 12)$, we see that

$$
\begin{aligned}
& \Gamma_{1, i}^{n+1} \circ \psi(0,0, t)=2 \operatorname{Im} K_{\overline{11} 1 \overline{1}}(0) \cdot t+O\left(t^{2}\right), \\
& \Gamma_{1, n+i}^{n+1} \circ \psi(0,0, t)=2 \operatorname{Re} K_{\overline{1} 1 \overline{1} i}(0) \cdot t+O\left(t^{2}\right) .
\end{aligned}
$$

Moreover, by (4) in Proposition $(2 \cdot 16)$ and $(2 \cdot 6)$, we see that $K_{\overline{1} 1 \overline{1} i}(0)$ $=0$. Hence we obtain

$$
\begin{aligned}
& V^{1}=\left\{-\frac{1}{l} \operatorname{Im} a_{1}+\frac{1}{l} \frac{\partial \operatorname{Im} \beta}{\partial \xi}\right\}+V_{1}^{(2)} \cdot t^{2}, \\
& V^{i}=V_{i}^{(1)} \cdot t \quad(i=2,3, \cdots, n), \\
& U^{i}=U_{i}^{(1)} \cdot t \quad(i=1,2, \cdots, n),
\end{aligned}
$$

where $V_{1}^{(2)}, V_{i}^{(1)}$ and $U_{i}^{(1)}$ are real analytic functions of $t$. By (3), (4) in Proposition $(2 \cdot 16)$, we see that

$$
\begin{aligned}
& \left|V_{1}^{(2)}\right| \leqq 2 n(n-1) C(2) C_{0}^{2} \delta_{0}^{-8}, \\
& \left|V_{i}^{(1)}\right| \leqq 2 n(n-1) C(1) C_{0}^{2} \delta_{0}^{-2} \quad(i=2,3, \cdots, n), \\
& \left|U_{1}^{(1)}\right| \leqq\left\{2 n(n-1) C(1) C_{0}{ }^{2}+2(n-1) C(1) C_{0}\right\} \delta_{0}{ }^{-2}, \\
& \left|U_{i}^{(1)}\right| \leqq 2 n(n-1) C(1) C_{0}^{2} \delta_{0}^{-2}(i=2,3, \cdots, n) .
\end{aligned}
$$

In the same manner as in A. Takeuchi [9, p. 343], we have

$$
\frac{\partial \beta}{\partial z^{i}}(0,0, \cdots, 0)=-2 g_{i, \overline{1}}(q) \quad \text { for } \quad i=2,3, \cdots, n .
$$

Therefore, by (4) in (ii), Lemma $(2 \cdot 10)$, we obtain

$$
\begin{aligned}
& \left|\frac{\partial \operatorname{Im} \beta}{\partial \xi}(0)-\operatorname{Im} a_{1}\right| \leqq 1+(n-1) C_{0}, \\
& \left|\frac{\partial \operatorname{Re} \beta}{\partial \xi}(0)-\operatorname{Im} a_{1}\right| \leqq 1+(n-1) C_{0} .
\end{aligned}
$$


Hence we obtain

$$
\int_{0}^{l} g\left(\nabla_{D_{1}} \widetilde{Y}, \nabla_{D_{1}} \widetilde{Y}\right) \circ \psi(0,0, t) d t=\frac{1}{l}\left(\frac{\partial \operatorname{Im} \beta}{\partial \xi}(0)-\operatorname{Im} a_{1}\right)^{2}+H(l) \cdot l^{2},
$$

where $|H(l)| \leqq M^{(2)}$ and $M^{(2)}$ is a constant which depends only on $C_{0}$ and $\hat{o}_{0}$.

Next we compute the third term in the left side of $(4 \cdot 10)$. By $(2 \cdot 2)$ and $(4 \cdot 7)$ and by using $\partial / \partial \xi\left(x^{i} \circ \psi\right)=Y^{i}$ and $\partial / \partial \xi\left(y^{i} \circ \psi\right)=Y^{n+i}$, $\nabla_{D_{2}} Y$ can be expressed as follows:

$$
\nabla_{D_{2}} Y=\sum_{i=1}^{n} V^{i} \frac{\partial}{\partial x^{i}} \circ \psi(0,0, t)+\sum_{i=1}^{n} W^{i} \frac{\partial}{\partial y^{i}} \circ \psi(0,0, t)
$$

where

$$
\begin{aligned}
V^{1} & =\frac{t}{l} \frac{\partial^{2} \operatorname{Re} \beta}{\partial \xi^{2}}+\sum_{i, j=1}^{2 n} Y^{i} Y^{j} \cdot \Gamma_{i, j}^{1} \circ \psi(0,0, t) \\
W^{1} & =\frac{t}{l} \frac{\partial^{2} \operatorname{Im} \beta}{\partial \xi^{2}}+\sum_{i, j=1}^{2 n} Y^{i} Y^{j} \cdot \Gamma_{i, j}^{n+1} \circ \psi(0,0, t) \\
V^{k} & =\sum_{i, j=1}^{2 n} Y^{i} Y^{j} \Gamma_{i, j}^{k} \circ \psi(0,0, t) \\
W^{k} & =\sum_{i, j=1}^{2 n} Y^{i} Y^{j} \Gamma_{i, j}^{n+k} \circ \psi(0,0, t)
\end{aligned}
$$

By $\Gamma_{j, 1}^{1} \circ \psi(0,0,0)=0$ and $\Gamma_{j, n+1}^{1} \circ \psi(0,0,0)=0$ for $j=1,2, \cdots, 2 n$ (see, (3), (4) in Proposition $(2 \cdot 15)$ and also $(2 \cdot 6)$ and $(2 \cdot 12))$, we have

$$
\begin{aligned}
g\left(\nabla_{D_{2}} Y, X\right) \circ & \left.\psi(0,0, t)\right|_{0} ^{l}=\frac{\partial^{2} \operatorname{Re} \beta}{\partial \xi^{2}}(0)+\sum_{i, j=2}^{n} \operatorname{Re} a_{i} \operatorname{Re} a_{j} \Gamma_{i, j}^{1} \circ \psi(0,0, l) \\
& +\sum_{i, j=2}^{n} \operatorname{Re} a_{i} \operatorname{Im} a_{j} \Gamma_{i, n+j}^{1} \circ \psi(0,0, l) \\
& +\sum_{i, j=2}^{n} \operatorname{Im} a_{i} \operatorname{Re} a_{j} \Gamma_{n+i, j}^{1} \circ \psi(0,0, l) \\
& +\sum_{i, j=2}^{n} \operatorname{Im} a_{i} \operatorname{Im} a_{j} \Gamma_{n+i, n+j}^{1} \circ \psi(0,0, l)+H^{\prime}(l) \cdot l^{2},
\end{aligned}
$$

where $\left|H^{\prime}(l)\right| \leqq M^{(3)}$ and $M^{(3)}$ is a positive constant which depends only on $C_{0}$ and $\delta_{0}$. This follows from (1), (2) and (4) in Proposition (2.16) and $(4 \cdot 11)$.

Hence we obtain 


$$
\begin{aligned}
& \frac{\partial^{2} D^{*}}{\partial \xi^{2}}(0,0)=\frac{1}{l}\left(\frac{\partial \operatorname{Im} \beta}{\partial \xi}-\operatorname{Im} a_{1}\right)^{2}+\frac{\partial^{2} \operatorname{Re} \beta}{\partial \xi^{2}}(0) \\
& \quad-\int_{0}^{l} g(R(Y, X) X, Y) \circ \psi d t \\
& \quad+\sum_{i, j=2}^{n} \operatorname{Re} a_{i} \operatorname{Re} a_{j} \Gamma_{i, j}^{1} \circ \psi(0,0, l)+\sum_{i, j=2}^{n} \operatorname{Re} a_{i} \operatorname{Im} a_{j} \Gamma_{i, n+j}^{1} \circ \psi(0,0, l) \\
& \quad+\sum_{i, j=2}^{n} \operatorname{Im} a_{i} \operatorname{Re} a_{j} \Gamma_{n+i, j}^{1} \circ \psi(0,0, l)+\sum_{i, j=2}^{n} \operatorname{Im} a_{i} \operatorname{Im} a_{j} \Gamma_{n+i, n+j}^{1} \circ \psi(0,0, l) \\
& \quad+H^{\prime \prime}(l) \cdot l^{2}
\end{aligned}
$$

where $\left|H^{\prime \prime}(l)\right| \leqq M^{(4)}$ and $M^{(4)}$ is a constant which depends only on $C_{0}$ and $\delta_{0}$.

In the same manner as above, we compute $\partial^{2} \Psi / \partial \eta^{2}(0,0)$ as follows

$$
\frac{\partial^{2} \Psi}{\partial \eta^{2}}(0,0)=\frac{1}{D^{* 2}}\left(\frac{\partial D^{*}}{\partial \eta}\right)^{2}(0,0)-\frac{1}{D^{*}} \frac{\partial^{2} D^{*}}{\partial \eta^{2}}(0,0),
$$

where

$$
\frac{\partial D^{*}}{\partial \eta}(0,0)=\left(\frac{\partial \operatorname{Re} \beta}{\partial \eta}+\operatorname{Im} a_{1}\right)+J(l) \cdot l^{3}
$$

and

$$
\begin{aligned}
& \frac{\partial^{2} D^{*}}{\partial \eta^{2}}(0,0)=\frac{1}{l}\left(\frac{\partial \operatorname{Im} \beta}{\partial \eta}-\operatorname{Re} a_{1}\right)^{2}+\frac{\partial^{2} \operatorname{Re} \beta}{\partial \eta^{2}}(0) \\
& \quad-\int_{0}^{l} g(R(Z, X) X, Z) \circ \psi d t \\
& \quad+\sum_{i, j=2}^{n}\left(-\operatorname{Im} a_{i}\right)\left(-\operatorname{Im} a_{j}\right) \Gamma_{i, j}^{1} \circ \psi(0,0, l) \\
& \quad+\sum_{i, j=2}^{n}\left(-\operatorname{Im} a_{i}\right)\left(\operatorname{Re} a_{j}\right) \Gamma_{i, n+j}^{1} \circ \psi(0,0, l) \\
& \quad+\sum_{i, j=2}^{n}\left(\operatorname{Re} a_{i}\right)\left(-\operatorname{Im} a_{j}\right) \Gamma_{n+i, j}^{1} \circ \psi(0,0, l) \\
& \quad+\sum_{i, j=2}^{n}\left(\operatorname{Re} a_{i}\right)\left(\operatorname{Re} a_{j}\right) \Gamma_{n+i, n+j}^{1} \circ \psi(0,0, l) \\
& \quad+J^{\prime}(l) \cdot l^{2},
\end{aligned}
$$

where $|J(l)| \leqq M^{(5)}$ and $\left|J^{\prime}(l)\right| \leqq M^{(6)}$ and $M^{(5)}, M^{(6)}$ are positive constants which depend only on $C_{0}$ and $\delta_{0}$. 
Therefore by using $(4 \cdot 11)$ and $\left|(\partial \operatorname{Re} \beta / \partial \eta)+\operatorname{Im} a_{1}\right| \leqq(n-1) C_{0}+1$ again, we obtain

$$
\begin{aligned}
& \Delta \Psi(0,0)=\frac{1}{l^{2}}\left\{\left(\frac{\partial D^{*}}{\partial \xi}(0,0)\right)^{2}+\left(\frac{\partial D^{*}}{\partial \eta}(0,0)\right)^{2}\right\}-\frac{1}{l}\left\{\frac{\partial^{2} D^{*}}{\partial \xi^{2}}(0,0)\right. \\
& \left.+\frac{\partial^{2} D^{*}}{\partial \eta^{2}}(0,0)\right\} \\
& =\frac{1}{l^{2}}\left\{\left(\frac{\partial \operatorname{Re} \beta}{\partial \xi}(0,0)-\operatorname{Re} a_{1}\right)^{2}+\left(\frac{\partial \operatorname{Re} \beta}{\partial \eta}(0,0)+\operatorname{Im} a_{1}\right)^{2}\right\} \\
& -\frac{1}{l^{2}}\left\{\left(\frac{\partial \operatorname{Im} \beta}{\partial \xi}(0,0)-\operatorname{Im} a_{1}\right)^{2}+\left(\frac{\partial \operatorname{Im} \beta}{\partial \eta}(0,0)-\operatorname{Re} a_{1}\right)^{2}\right\} \\
& -\frac{1}{l}\left\{\left(\frac{\partial^{2} \operatorname{Re} \beta}{\partial \xi^{2}}(0,0)+\frac{\partial^{2} \operatorname{Re} \beta}{\partial \eta^{2}}(0,0)\right\}\right. \\
& -\frac{1}{l}\left\{\sum_{i, j=2}^{n} \operatorname{Re} a_{i} \operatorname{Re} a_{j}\left(\Gamma_{i, j}^{1}+\Gamma_{n+i, n+j}^{1}\right) \circ \psi(0,0, l)\right. \\
& +\sum_{i, j=2}^{n} \operatorname{Re} a_{i} \operatorname{Im} a_{j}\left(\Gamma_{i, n+j}^{1}-\Gamma_{n+i, j}^{1}\right) \circ \psi(0,0, l) \\
& +\sum_{i, j=2}^{n} \operatorname{Im} a_{i} \operatorname{Re} a_{j}\left(\Gamma_{n+i, j}^{1}-\Gamma_{i, n+j}^{1}\right) \circ \psi(0,0, l) \\
& \left.+\sum_{i, j=2}^{n} \operatorname{Im} a_{i} \operatorname{Im} a_{j}\left(\Gamma_{n+i, n+j}^{1}+\Gamma_{i, j}^{1}\right) \circ \psi(0,0, l)\right\} \\
& +\frac{1}{l} \int_{0}^{l}\{g(R(Y, X) X, Y)+g(R(Z, X) X, Z)\} \circ \phi(0,0, t) d t \\
& +M(l) \cdot l,
\end{aligned}
$$

where $|M(l)| \leqq M^{(7)}$ and $M^{(7)}$ is a positive constant which depends only on $C_{0}$ and $\delta_{0}$.

Because $\beta$ is a holomorphic function, we see that

$$
\frac{\partial \operatorname{Re} \beta}{\partial \xi}=\frac{\partial \operatorname{Im} \beta}{\partial \eta} \text { and } \frac{\partial \operatorname{Re} \beta}{\partial \eta}=-\frac{\partial \operatorname{Im} \beta}{\partial \xi} \text {. }
$$

Also by $(2 \cdot 12)$, we have

$$
\Gamma_{i, j}^{1}+\Gamma_{n+i, n+j}^{1}=0 \text { and } \Gamma_{n+i, j}^{1}-\Gamma_{i, n+j}^{1}=0 .
$$

Hence all the terms except the last two ones cancel out. Thus we obtain 
Lemma (4-12). There exists a positive constant $M^{(7)}$ which depends only on $C_{0}$ and $\delta_{0}$ such that

$$
\begin{aligned}
\Delta \Psi(0,0)= & \frac{1}{l} \int_{0}^{l}\{g(R(Y, X) X, Y)+g(R(Z, X) X, Z)\} \circ \psi(0,0, t) d t \\
& +F(l) \cdot l
\end{aligned}
$$

where $|F(l)| \leqq M^{(7)}$.

By $(2 \cdot 8)$ and $(4 \cdot 8)$, we have

$$
\begin{gathered}
\int_{0}^{l}\{g(R(Y, X) X, Y)+g(R(Z, X) X, Z)\} \circ \psi(0,0, t) d t \\
\geq \rho\left[\int_{0}^{l}\left\{g(X, X) g(Y, Y)-g(X, Y)^{2}\right\} \circ \psi(0,0, t) d t\right. \\
\left.\quad+\int_{0}^{l}\left\{g(X, X) g(Z, Z)-g(X, Z)^{2}\right\} \circ \psi(0,0, t) d t\right] .
\end{gathered}
$$

The integrands in the left side can be estimated as follows: By $g(X, X)$ $=1$, we have

$$
\begin{aligned}
& \left\{g(X, X) g(Y, Y)-g(X, Y)^{2}\right\} \circ \psi(0,0, t) \\
& \quad=\left\{\left(1-\frac{t}{l}\right) \operatorname{Im} a_{1}+\frac{t}{l} \frac{\partial \operatorname{Im} \beta}{\partial \xi}\right\}^{2}+\sum_{i=2}^{n}\left(\operatorname{Re} a_{i}\right)^{2}+\sum_{i=2}^{n}\left(\operatorname{Im} a_{i}\right)^{2}+H(t) \cdot t^{2},
\end{aligned}
$$

where $|H(t)| \leqq M^{(8)}$ and $M^{(3)}$ is a positive constant which depends only on $C_{0}$ and $\delta_{0}$. In the same manner, we have

$$
\begin{aligned}
& \left\{g(X, X) g(Z, Z)-g(X, Z)^{2}\right\} \circ \psi(0,0, t) \\
& \quad=\left\{\left(1-\frac{t}{l}\right) \operatorname{Re} a_{1}+\frac{t}{l} \frac{\partial \operatorname{Im} \beta}{\partial \eta}\right\}^{2}+\sum_{i=2}^{n}\left(\operatorname{Re} a_{i}\right)^{2}+\sum_{i=2}^{n}\left(\operatorname{Im} a_{i}\right)^{2}+H^{\prime}(t) \cdot t^{2},
\end{aligned}
$$

where $\left|H^{\prime}(t)\right| \leqq M^{(9)}$ and $M^{(9)}$ is a positive constant which depends only $C_{0}$ and $\delta_{0}$. By

$$
\begin{aligned}
& \int_{0}^{l}\left\{\left(1-\frac{t}{l}\right) \operatorname{Im} a_{1}+\frac{t}{l} \frac{\partial \operatorname{Im} \beta}{\partial \xi}\right\}^{2} d t \geqq \frac{l}{4}\left(\operatorname{Im} a_{1}\right)^{2}, \\
& \int_{0}^{l}\left\{\left(1-\frac{t}{l}\right) \operatorname{Re} a_{1}+\frac{t}{l} \frac{\partial \operatorname{Im} \beta}{\partial \eta}\right\}^{2} d t \geqq \frac{l}{4}\left(\operatorname{Re} a_{1}\right)^{2},
\end{aligned}
$$

the right side of $(4 \cdot 13)$ can be bounded below by the following: 


$$
\frac{\rho}{4}+H^{\prime \prime}(l) \cdot l^{2}
$$

where $\left|H^{\prime \prime}(l)\right| \leqq M^{(10)}$ and $M^{(10)}$ is a positive constant which depends only on $C_{0}$ and $\delta_{0}$.

Finally we obtain

$$
\Delta \Psi(0,0) \geqq \frac{\rho}{4}+H^{\prime \prime \prime}(l) \cdot l,
$$

where $\left|H^{\prime \prime \prime}(l)\right| \leqq M^{*}$ and $M^{*}$ is a positive constant which depends only on $C_{0}$ and $\delta_{0}$.

By $(4 \cdot 14)$ we prove our Key Lemma. We choose $\delta^{*}$ by the following condition:

$$
\delta^{*}=\min \left(\rho / 8 M^{*}, \delta^{\prime}\right) .
$$

Take an arbitrary point $p \in D_{\delta^{*}}$. Then as we stanted in the beginning of this section, we can find a point $q \in \partial D$ such that $d(p)=d(p, q)$ and a geodesic between $p$ and $q$, which is denoted by $\sigma$. We choose a system of local coordinates $z^{1}, z^{2}, \cdots, z^{n}$ on $U_{\delta_{0}(i)}(z: p)$ as in (ii) in Lemma $(2 \cdot 10)$ and a divisor $S$ through $q$ as in Proposition (3.3). And for any complex line $N$, we construct a holomorphic variation $\psi$ of $\sigma$ as $(4 \cdot 3)$. Then for any point $r \in N$, we see that

$$
d(r) \leqq D^{*}(r) \text { and } d(p)=D^{*}(p) .
$$

By the choice of $\delta^{*}$,

$$
\Delta \Psi(p) \geqq \rho / 8
$$

In view of $\varphi(r)=-\log d(r)$ and $\Psi(r)=-\log D^{*}(r)$, we have

$$
\varphi(r) \geqq \Psi(r) \text { and } \varphi(p)=\Psi(p) .
$$

Therefore by $(3 \cdot 5)$, we have

$$
\Delta \varphi(p) \geqq \Delta \Psi(p),
$$

which proves by $(3 \cdot 1)$ that

$$
W(\varphi(p)) \geqq \rho / 8 \text { for } p \in D_{\delta^{*}} .
$$

This completes the proof. 


\section{$\S 5$. Proof of Main Theorem}

Finally we prove our Main Theorem. Let $D$ be a general pseudoconvex domain on $M$ and let $C_{0}$ be a positive constant with $C_{0}>1$. Take an arbitrary point $p \in \partial D$ and a geodesic through $p$. Then by (i) in Lemma $(2 \cdot 10)$ we can choose neighborhoods, $V(p)$ and $U_{\hat{0}_{0}}(p)$. Making $V(p)$ smaller, we may assume that Proposition (3.4) holds for $V(p)$ and $U_{\delta_{0}}(p)$. By using (i) in Lemma $(2 \cdot 10)$ again, we choose $V(p)$ and $U_{\hat{\delta}_{0}}(p)$ for every $p \in \bar{D}$, where we assume that especially when $p \in \partial D$, $V(p)$ satisfies the above condition. Then there exist $\left\{p_{i}\right\} \quad i=1,2, \cdots, N$ such that $\cup_{i=1}^{N} V\left(p_{i}\right) \supset \bar{D}$. Then setting $\delta=\min _{i=1,2, \ldots, N} \delta^{(i)}$ where $\delta^{(i)}$ is chosen as $\delta$ in (i) in Lemma $(2 \cdot 10)$ at $p_{i}$, we see that $\delta, \delta_{0}^{(1)}, \delta_{0}{ }^{(2)}, \cdots, \delta_{0}{ }^{(N)}$ satisfy (ii) in Lemma $(2 \cdot 10)$, where $\delta_{0}{ }^{(i)}$ is chosen as $\delta_{0}$ in (i) at $p_{i}$. Here we may assume that there exists a subset $\left\{p_{j}\right\}_{j=1,2, \ldots, r}$ such that every $p_{j}$ is contained in $\partial D$ and $\cup_{j=1}^{r} V\left(p_{j}\right) \supset \partial D$. By $M^{*}$ we denote the positive constant which is determined by $C_{0}$ and $\delta_{0}$ in $(4 \cdot 14)$. We define $\delta^{*}$ by

$$
\delta^{*}=\min \left(\rho / 8 M^{*}, \delta^{\prime}\right),
$$

where $\delta^{\prime}$ is defined by the same condition as in the beginning of $\S 4$. Making $\delta^{*}$ smaller, we may assume that $D_{0^{*}} \subset \bigcup_{i=1}^{r} V\left(p_{i}\right)$. Choosing a point $p \in D_{0^{\star}}$. We assume $p \in V\left(p_{i}\right)$. As in $\S 3$, we choose a sequence of domains $\left\{A_{j}\right\}$ of $U_{\delta_{0}(i)}(Z: p) \cap D$. Then there exists an integer $j_{0}$ such that $p \in \Delta_{j}$ for $j \geqq j_{0}$. As (3.5) we define $d_{j}(r)$ and $\varphi_{j}(r)$ for $r \in \Delta_{j_{0}}$. By the choice of $\delta^{*}$, we see that $d_{j}(r)<\delta^{*}$ for $r \in \Delta_{j_{0}} \cap D_{\delta^{*}}$. So by Key Lemma, we find that

$$
W\left(\varphi_{j}(p)\right) \geqq \rho / 8 .
$$

As in the end of $\S 3$, we see that $\varphi_{i}(p)>\varphi_{i+1}(p)$ for $i \geqq j_{0}$ and $\varphi_{j}(p) \rightarrow$ $\varphi(p)$. Therefore we obtain

$$
W(\varphi(p)) \geqq \rho / 8,
$$

which implies

$$
W(\varphi(p)) \geqq \rho / 8 \text { for } p \in D_{\delta^{*}} .
$$

Then By Theorem (3.2), we prove Main Theorem. 


\section{References}

[1] Fujita, R., Domains sans point critique intérieur sur l'espace projectif complexe, J. Math. Soc. Japan, 15 (1963), 443-473.

[2] Goldberg, S. I. and Kobayashi, S., On holomorphic bisectional curvature, $J$. Differential Geometry, 1 (1967), 225-233.

[3] Grauert, H., Charakterisierung der Holomorphiegebiete durch die vollstäntige Kählershe Metrik, Math. Ann., 131 (1956), 38-75.

[4] - On Levi's problem and the embedding of real analytic manifolds, Ann. of Math., 68 (1958), 460-472.

[5] - Über Modifikationen und exzeptionelle analytische Mengen, Math. Ann., 146 (1962), 331-368.

[6] Gromoll, D., Klingenberg, W. and Meyer, W., Riemannsch Geometrie im Großen, Lecture notes in Mathematics 55, Springer, 1968.

[7] Kobayashi, S. and Nomizu, K., Foundations of Differential Geometry, I, II, Interscience Publishers, New York, 1963, 1969.

[8] Takeuchi, A., Domains pseudoconvexes sur les variétés kählériennes, J. Math. Kyoto Univ., 6 (1967), 323-357.

Note added in proof. After this paper was submitted for publication, the auther was foformed from Mr. T. Sasaki that the paper of G. Elencwajg "Pseuto-convexite locale dans les variétés kahlériennes" appeared in Ann. Inst. Fourier (Grenoble), 25 (1975), 295-314. In the forthcomming paper in this journal, the auther will prove an analogous theorem in tee case of $C^{\infty}$-kähler metrics. 